\title{
Several Semiclassical Approaches to Time-resolved Spectroscopy
}

\author{
Jiří Vaníček*
}

\begin{abstract}
Ultrafast spectroscopy allows molecular dynamics to be resolved on the femtosecond time scale. Whereas such short time scales obviously pose many experimental challenges, they provide an opportunity for semiclassical methods, which are naturally suited for short time dynamics. Here we review several semiclassical approaches for evaluating vibrationally resolved electronic pump-probe spectra, starting with the simplest, 'phase averaging' or 'dephasing representation'. We continue by discussing several methods developed in our group that allow increasing the efficiency (the cellular dephasing representation) and accuracy (cellular dephasing representation with a prefactor) and end with the Gaussian dephasing representation, which, despite its semiclassical origins, converges to the exact quantum result. The merits as well as shortcomings of the different approaches are demonstrated on time-resolved stimulated emission spectra of NCO and pyrazine.
\end{abstract}

Keywords: Dephasing representation · Fidelity amplitude · Phase averaging · Semiclassical approximation . Time correlation function · Time-resolved stimulated emission

\section{Introduction}

High time resolution (such as $10^{-12} \mathrm{~s}$ or even $10^{-15} \mathrm{~s}$ ) is important for understanding physical and chemical processes induced by the interactions of molecules with light; indeed, the femtosecond time resolution has been the main challenge of ultrafast spectroscopy for almost three decades. In contrast to the experimental difficulties, one expects that the short time scales should simplify theoretical simulations by requiring shorter propagation of the molecular wavepacket. Yet, whenever nuclear quantum effects play an essential role, even short-time simulations of the time-dependent Schrödinger equation are difficult because of their exponential scaling with the number of degrees of freedom.

To make such calculations practical, it is necessary to develop approximate dynamical methods, which are feasible with the computational resources available and,

${ }^{\star}$ Correspondence: Prof. Dr. J. Vaníček Ecole Polytechnique Federale de Lausanne (EPFL) Institut des Sciences et Ingenierie Chimiques Laboratory of Theoretical Physical Chemistry EPFL SB ISIC LCPT, BCH 3110

$\mathrm{CH}-1015$ Lausanne

E-mail: jiri.vanicek@epfl.ch at the same time, sufficiently accurate to answer the questions of interest. In the case of continuous-wave spectroscopy, where the light is not pulsed, but its coupling to the molecular motion is moderately weak so that the first-order time-dependent perturbation theory is valid, a very useful picture of molecule-light interaction in terms of wavepacket autocorrelation functions has been developed already in the 1970s and 1980s, especially by Heller, ${ }^{[1]}$ who also suggested a very simple semiclassical approximation, ${ }^{[2]}$ now called the thawed Gaussian approximation, to evaluate various types of electronic spectra.

In the field of ultrafast spectroscopy, one must invoke higher orders of the timedependent perturbation theory, a reward for this effort being an even richer variety of phenomena. A systematic analysis of correlation functions and response functions contributing to various types of timeresolved spectra has been developed in the 1980s and 1990s, and is summarized in a comprehensive book ${ }^{[3]}$ by Mukamel, who also proposed a very simple semiclassical method, called phase averaging, ${ }^{[4]}$ allowing the evaluation of various types of timeresolved spectra.

In this article, we review several recently developed semiclassical methods for evaluating time-resolved electronic spectra that can be thought of as extensions of Mukamel's phase averaging. Starting from an alternative presentation of linear spectroscopy that makes the analogy to nonlinear spectroscopy obvious, we describe the phase averaging, the dephasing representation and its several variants that can make the method more efficient, more accurate, and sometimes even exact.

\section{Time-dependent Approach to Spectroscopy}

\subsection{Linear Spectra: Autocorrelation Function vs. Fidelity Amplitude}

In the time-dependent approach to spectroscopy, pioneered by Heller, ${ }^{[1]}$ the linear electronic absorption spectrum $\sigma(\omega)$ of a molecule can be computed as the Fourier transform

$$
\sigma(\omega)=\frac{4 \pi \omega}{3 h c} \mu_{01}^{2} \operatorname{Re} \int_{0}^{\infty} C(t) e^{i\left(\omega+E_{0,0} / \hbar\right) t} d t
$$

of the wavepacket autocorrelation function

$$
C(t)=\langle\psi \mid \psi(t)\rangle=\left\langle\psi\left|e^{-i \hat{H}_{1} t / \hbar}\right| \psi\right\rangle
$$

of an initial state $|\psi\rangle \equiv|0,0\rangle$ given by the vibrational ground state of the electronic ground state potential energy surface, moving on the excited state potential energy surface described by the Hamiltonian operator $\hat{H}_{1}$. Here $\mu_{01}$ is the transition dipole moment between the ground and excited electronic states, $\omega$ is the frequency of the electromagnetic radiation, and $E_{0,0}$ denotes the zero point vibrational energy in the ground electronic state. Eqn. (1) assumes the validity of the electric dipole approximation (requiring the wavelength of the electromagnetic field to be much larger than the size of the molecule), the first-order time-dependent perturbation theory (restricting the strength of the electromagnetic field), Condon approximation (requiring the transition dipole to be independent of the nuclear coordinates), and low temperature approximation (implying that only the ground vibrational 
state $|0,0\rangle$ of the ground surface be occupied initially).

The beauty of Eqns. (1) and (2) lies in their simple interpretation (see also Fig. 1 (a)): the absorption of a photon of frequency $\omega$ instantaneously promotes the stationary vibrational ground state of the ground surface to the excited potential energy surface, where this, now nonstationary state starts moving under the influence of the excited state Hamiltonian alone. In particular, the explicit form of the electromagnetic field does not play any role and the linear absorption spectrum is determined solely by the field-free dynamics of the wavepacket $\psi(t)$ on the excited surface. Indeed, this is the content of the linear response theory, which is here equivalent to the first-order time-dependent perturbation theory.

Note that since $|\psi\rangle \equiv|0,0\rangle$ is an eigenstate of $\hat{H}_{0}$, the spectrum can be also written as

$$
\sigma(\omega)=\frac{4 \pi \omega}{3 h c} \mu_{01}^{2} \operatorname{Re} \int_{0}^{\infty} f(t) e^{i \omega t} d t,
$$

where

$$
f(t)=\left\langle\psi_{0}(t) \mid \psi_{1}(t)\right\rangle=\left\langle\psi\left|e^{i \hat{H}_{0} t / \hbar} e^{-i \hat{H}_{1} t / \hbar}\right| \psi\right\rangle(4)
$$

is a correlation function, called the fidelity amplitude, ${ }^{[5]}$ between two states $\psi_{0}(t)$ and $\psi_{1}(t)$, both starting from the same initial state $\psi$, but one evolved with $\hat{H}_{0}$ and the other with $\hat{H}_{1}$. As the name suggests, the fidelity amplitude measures the similarity between the quantum evolutions on the ground and excited surfaces. This alternative expression for an electronic spectrum is not a mathematical curiosity; indeed, it is the direct outcome of the derivation of the spectrum using the first-order time-dependent perturbation theory, and it is only due to the additional assumption that $\psi$ is a vibrational ground state (or another eigenstate) of $\hat{H}_{0}$ that one obtains the much better known expression (1) for the spectrum in terms of the wavepacket autocorrelation function (2).

The less often used correlation function (4) has, nonetheless, many important

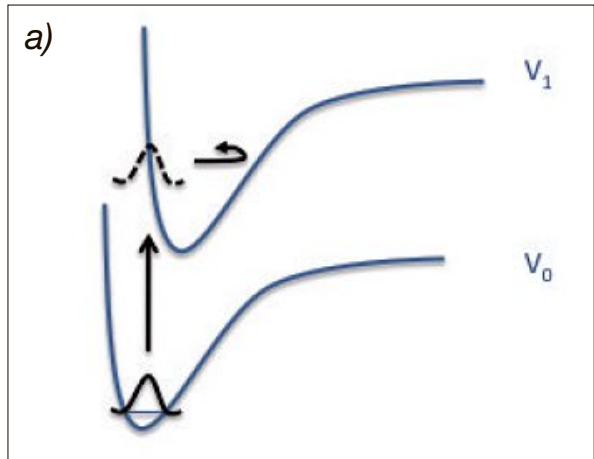

Fig. 1. Schematic representation of physical processes underlying two types of vibrationally resolved electronic spectra. (a) Linear absorption. (b) Time-resolved stimulated emission. applications: Outside of electronic spectroscopy, ${ }^{[6-12]}$ it has proved useful, e.g. in NMR spin echo experiments ${ }^{[13]}$ and theories of quantum computation, ${ }^{[5]}$ decoherence, ${ }^{[5]}$ and inelastic neutron scattering. ${ }^{[14]}$ In chemical physics, the fidelity amplitude was also used as a measure of the dynamical importance of diabatic, ${ }^{[15]}$ nonadiabatic, ${ }^{[16]}$ or spin-orbit couplings, ${ }^{[17]}$ and of the accuracy of quantum molecular dynamics on an approximate potential energy surface. ${ }^{[18,19]}$

\subsection{Time-resolved Electronic Spectra}

In the case of nonlinear spectra, the autocorrelation picture is no longer valid, yet, as we now show, the more general picture using fidelity amplitude remains applicable. A wide variety of nonlinear timeresolved spectra belong to the pump-probe scheme, in which an ultrashort pump pulse prepares a nonstationary nuclear wavepacket in an excited electronic state, and another ultrashort pulse probes the dynamics of this wavepacket after a certain time delay $\tau$. There are many possible experimental setups depending on the polarization and mutual orientation of the pump and probe laser beams and on the direction in which the signal is detected, ${ }^{[3]}$ but for the sake of clarity we will only consider timeresolved stimulated emission (TRSE) here (see Fig. 1 (b)).

Besides the assumptions used for linear spectra, a simplified picture of TRSE takes advantage of the nonoverlapping pulses approximation (i.e. the pump and probe pulses can be treated independently) and the ultrashort pulse approximation, which assumes that both the pump and probe pulses are short compared to the nuclear time scale but long on the electronic time scale.

Assuming, for simplicity and as before, the zero temperature approximation, electric dipole approximation, and timedependent perturbation theory (of which the third order is now required), the differential TRSE spectrum at frequency $\omega$ and time delay $\tau$ can be computed as the Fourier transform

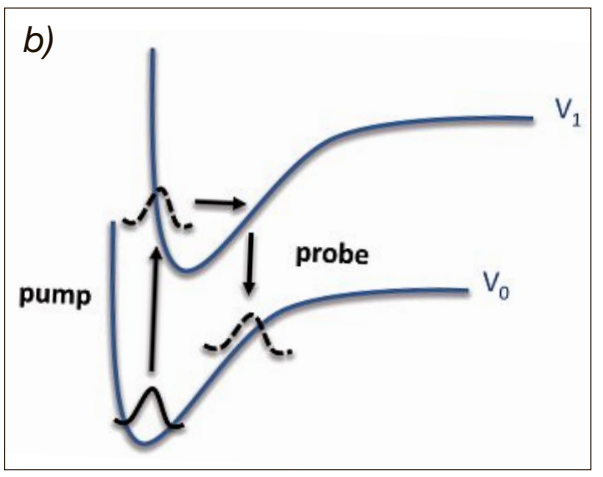

$\sigma(\omega, \tau) \propto \operatorname{Re} \int_{0}^{\infty} f(t, \tau) e^{i \omega t} d t$

of the wavepacket correlation function $^{[6,20,21]}$

$f(t, \tau)=\left\langle\psi_{1}(t, \tau) \mid \psi_{0}(t, \tau)\right\rangle$,

where $\tau$ is the time delay between the pump and probe pulses, $t$ denotes the time elapsed after the probe pulse, and

$$
\left|\psi_{j}(t, \tau)\right\rangle:=e^{-i \hat{H}_{j} t / \hbar} e^{-i \hat{H}_{1} \tau / \hbar}|\psi\rangle
$$

stands for the initial state evolved for the delay time $\tau$ with the excited state Hamiltonian and subsequently for time $t$ with either the ground or excited state Hamiltonian $(j=0,1)$.

As written, the correlation function $f(t, \tau)$ from Eqn. (6) has an immediate interpretation as a quantum fidelity amplitude between states $\psi_{0}(t, \tau)$ and $\psi_{1}(t, \tau)$. This fidelity amplitude now corresponds to evolutions for time $t+\tau$ of the same initial state $\psi$ with two Hamiltonians, one Hamiltonian being time-independent and equal to $\hat{H}_{1}$, the other becoming time-dependent and given by $\hat{H}_{1}$ until time $\tau$, and by $\hat{H}_{0}$ at later times. Note that the correlation function $f(t, \tau)$ can be also interpreted as a correlation function (4) from linear spectroscopy, but applied to a nonstationary initial state $\exp \left(-i \tau \widehat{H}_{1} / \hbar\right)|\psi\rangle$ prepared by the pump pulse. ${ }^{[6,7]}$

\section{Several Semiclassical Approaches to Time-resolved Electronic Spectra}

Correlation functions $C(t), f(t)$, or $f(t, \tau)$ can of course be evaluated exactly quantum mechanically, but this requires the exact solution of the time-dependent Schrödinger equation, which can be prohibitively expensive in many dimensions. A nice feature of electronic spectra, and ultrafast spectra in particular, is their short-time nature, which offers itself to approximative treatment of dynamics. Semiclassical approximation provides a perfect candidate since it is typically exact for short times while its accuracy deteriorates at longer times. As the simplest starting point, Heller originally used a single Gaussian as an ansatz for the wavefunction, which is exact in up to quadratic potentials and yields the thawed Gaussian approximation in general potentials. ${ }^{[2]}$ Alternatively, one may employ multiple trajectory-based methods such as the frozen Gaussian approximation, ${ }^{[22]}$ initial value representation, ${ }^{[23,24]}$ or their 
combination giving the Herman-Kluk propagator, ${ }^{[25]}$ but these methods become quickly expensive since they require both the Hessian of the potential energy surface and very large numbers of trajectories for convergence. Here we will review several variants of an alternative, very simple semiclassical approximation, called phase averaging, dephasing representation, or Wigner averaged classical limit, which require only the gradient of the potential energy, a rather small number of trajectories for convergence, and take advantage of the specific form of the correlation functions corresponding to electronic spectra.

\subsection{Phase-averaging/Dephasing Representation}

A remarkably simple approximation for the correlation function (or fidelity amplitude) $f(t)$ is given by the so-called phase averaging, dephasing representation, or Wigner averaged classical limit ${ }^{[3,4,7-12,26,27]}$ :

$$
f_{\mathrm{DR}}(t)=h^{-D} \int d x_{0} \rho_{\mathrm{W}}\left(x_{0}\right) e^{i \Delta S\left(x_{0}, t\right) / \hbar},
$$

where $D$ is the number of degrees of freedom, $\rho_{\mathrm{W}}\left(x_{0}\right)$ is the Wigner phase-space representation of the initial state $\psi$ and

$$
\Delta S\left(x^{0}, t\right)=-\int_{0}^{t} d t^{\prime} \Delta V\left(x_{t^{\prime}}\right)
$$

is the action due to the difference $\Delta V:=$ $V_{1}-V_{0}$ between the two potential energy surfaces along the classical trajectory $x$ $\equiv\left(q_{t}, p_{t}\right)$ driven by the average ${ }^{[3,4,20,28]}$ Hamiltonian $\bar{H} \equiv\left(H_{0}+H_{1}\right) / 2$.

In the original phase averaging, ${ }^{[4]}$ the weight function in Eqn. (8) was a classical density $\rho\left(x_{0}\right)$, and three options for the Hamiltonian used for driving the trajectories were considered: besides $\bar{H}$, one could use $H_{1}$ (suitable for absorption spectra) or $H_{0}$ (suitable for emission spectra). Replacement of the classical density with the more accurate Wigner function gave rise to the name 'Wigner averaged classical limit'. ${ }^{[10,11]}$ The name 'dephasing representation', on the other hand, suggests that the overlap of $\psi_{0}$ and $\psi_{1}$ in this approximation decays only due to dephasing, i.e. a destructive interference, whereas the classical overlap is assumed to be constant and fixed at 1 . The dephasing representation becomes applicable to ultrafast spectra after an appropriate generalization of the fidelity amplitude. In the case of TRSE spectra (Eqns. (5) and (6)), the fidelity amplitude can be approximated as

$$
f_{\mathrm{DR}}(t, \tau)=h^{-D} \int d x_{0} \rho_{\mathrm{W}}\left(x_{0}\right) e^{i \Delta S\left(x_{0}, \tau, t\right) / \hbar},
$$

where the action difference is given by

$$
\Delta S\left(x^{0}, \tau, t\right)=\int_{\tau}^{\tau+t} d t^{\prime} \Delta V\left(x_{t^{\prime}}\right)
$$

and the trajectory $x_{t}$, follows the excited state Hamiltonian $H_{1}$ for $0<t^{\prime}<\tau$ and the average Hamiltonian $\bar{H}$ for $t^{\prime}>\tau$. (From now on, for brevity we shall only present expressions for the TRSE spectra, and no more for linear absorption spectra.)

Expression (10) immediately suggests a numerical recipe for its evaluation: 1) sample initial conditions $x_{0}$ from the phase space density $\rho_{\mathrm{w}}\left(x_{0}\right), 2$ ) run classical trajectories with these initial conditions, 3 ) evaluate the phase (11) due to the difference of the potential energies along each of these trajectories, and 4) average over these trajectories. In a more compactform, this recipe can be expressed as

$$
f_{\mathrm{DR}}(t, \tau)=\left\langle e^{i \Delta S\left(x_{0}, \tau, t\right) / \hbar}\right\rangle_{\rho_{\mathrm{W}}\left(x_{0}\right)},
$$

where $\left\langle A\left(x_{0}\right)\right\rangle_{\rho(x 0)}$ denotes, more generally, an average of an observable $A\left(x_{0}\right)$ over initial conditions $x_{0}$ sampled from the density $\rho\left(x_{0}\right)$.

There are many ways to derive the dephasing representation: it can be obtained, ${ }^{[26,27]}$ e.g. by linearizing the semiclassical propagator, which is a procedure inspired by the semiclassical perturbation theory. ${ }^{[29,30]}$ Shi and Geva ${ }^{[12]}$ derived the same approximation (but referred to it as the linearized semiclassical initial value representation) without invoking the semiclassical propagator - by linearizing directly the path integral quantum propagator. Among the appeals of the dephasing representation is the ease with which it is numerically evaluated: We have shown ${ }^{[31]}$ that the expected number of trajectories required for convergence of the dephasing representation is independent of dimensionality, time, and nature of the dynamics, and depends explicitly only on the magnitude of the correlation function one wants to simulate.

\subsection{Increasing the Efficiency and Accuracy: Cellular Dephasing Representation with a Prefactor}

Unlike other semiclassical methods, which typically require a Hessian of the potential energy and thousands or millions of trajectories for convergence, the dephasing representation only needs the energy gradient, and about hundred to thousand trajectories for full convergence. This extraordinary efficiency of the dephasing representation makes it a promising candidate for on-the-fly $a b$ initio evaluation of time-resolved spectra.

Yet, for large systems, even a thousand trajectories may be too much to ask for, and hence we have attempted to reduce the number of trajectories by so-called cellularization, inspired by cellular dynamics ${ }^{[32]}$ and Filinov filtering ${ }^{[33-35]}$ used for other semiclassical methods. In the cellular dephasing representation (CDR), ${ }^{[21,36]}$ the initial conditions are grouped into cells of neighboring trajectories, and contributions of all trajectories within a cell are evaluated approximately analytically using the information collected along the central trajectory of the cell. This leads only to a minor modification of the numerical algorithm (12), which becomes

$$
f_{\mathrm{CDR}}(t, \tau)=\left\langle A_{\mathrm{CDR}} e^{i \Delta S\left(x_{0}, \tau, t\right) / \hbar}\right\rangle_{\rho_{\mathrm{IWT}}\left(x_{0}\right)},
$$

where $\mathrm{A}_{\mathrm{CDR}}\left(x_{0}, \tau, t\right)$ is a prefactor ${ }^{[36]}$ capturing the contribution of neighboring trajectories and $\rho_{\mathrm{IWT}}$ is the inverse Weierstrass transform of $\rho_{\mathrm{W}}$ (in other words, it is the expansion coefficient of $\rho_{\mathrm{w}}$ in a basis of phase space Gaussians). ${ }^{[36]}$

Fig. 2 shows that in a two-dimensional model of $\mathrm{NCO},{ }^{[21]}$ which is only slightly anharmonic, the cellular dephasing representation based on a single trajectory can yield a time-resolved stimulated emission spectrum that agrees very closely with the fully converged dephasing representation (using over sixteen thousand trajectories).

Although the dephasing representation is exact for displaced harmonic oscillators $^{[3]}$ and remarkably accurate in chaotic systems, ${ }^{[26]}$ its efficiency, of course, does not come for free. Due to its relation to the semiclassical perturbation approximation, the dephasing representation breaks down even for only quadratic perturbations encountered already in harmonic systems with different force constants. Unfortunately, these are important in so-called 'silent modes' in electronic spectroscopy, which are modes that are not vibrationally excited by the electronic excitation; if there

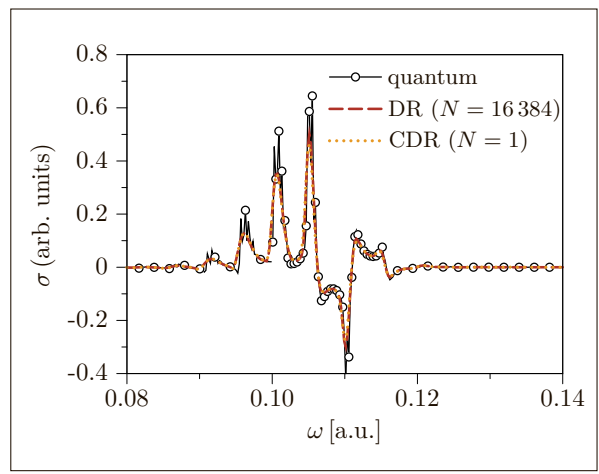

Fig. 2. Time-resolved stimulated emission spectrum of a collinear model of NCO from ref. [21] for a delay time $\tau=500$ a.u. $\approx 12$ fs. The spectrum obtained with the cellular dephasing representation (CDR) using a single trajectory (!) is in remarkable agreement with the fully converged spectrum obtained with the original dephasing representation (DR, computed using $N=16384$ trajectories), which, in turn, reproduces the main qualitative features of the exact quantum spectrum. (Adapted from ref. [21].) 
are many such 'boring' modes, the dephasing representation breaks down completely due to an artificially fast decay of the correlation function. Zambrano and Ozorio de Almeida proposed ${ }^{[28]}$ a simple recipe for partially correcting this inaccuracy by including a (different) prefactor $\mathrm{A}_{\mathrm{DRP}}\left(x_{0}, \tau, t\right)$ in the dephasing representation, resulting in the dephasing representation with $a$ prefactor (DRP):

$$
f_{\mathrm{DRP}}(t, \tau)=\left\langle A_{\mathrm{DRP}} e^{i \Delta S\left(x_{0}, \tau, t\right) / \hbar}\right\rangle_{\rho_{\mathrm{W}}\left(x_{0}\right)} .
$$

It turns out that the two prefactors in Eqns (13) and (14) can be easily combined, yielding the cellular dephasing representation with a prefactor (CDRP), ${ }^{[36]}$ which may be both more accurate and more efficient than the original dephasing representation:

$$
f_{\mathrm{CDRP}}(t, \tau)=\left\langle A_{\mathrm{CDR}} A_{\mathrm{DRP}} e^{i \Delta S\left(x_{0}, \tau, t\right) / \hbar}\right\rangle_{\rho_{\mathrm{IWT}}\left(x_{0}\right)} .
$$

Indeed, Fig. 3 shows on the timeresolved stimulated emission of pyrazine that the cellular dephasing representation with a prefactor is not only more accurate but also requires fewer trajectories for convergence than does the original dephasing representation. (Note, however, that this property is not universal, and that in strongly chaotic systems, such as the quartic oscillator, the original dephasing representation can converge faster than the CDRP, since a few chaotic trajectories can result in large prefactors that require many well-behaved trajectories to compensate this blowup in the final result.[36]) Even if the number of trajectories is reduced by the cellularization, the cost of each trajectory increases significantly since the prefactors $A_{\mathrm{CDR}}$ and $A_{\mathrm{DRP}}$ require the evaluation of the Hessian or even the third derivatives of the potential energy, unlike the dephasing representation for which the force is all that is needed.

\subsection{Making the Dephasing Representation Exact: Gaussian Dephasing Representation}

An alternative approach for improving the accuracy of the dephasing representation replaces the swarm of $N$ independent semiclassical trajectories with a swarm of $N$ 'communicating' Gaussian basis functions moving along corresponding classical trajectories. This trick is closely related to the basic idea employed in multiple spawning, [37] variational Gaussian wavepackets, ${ }^{[38]}$ coupled coherent states, and multiconfigurational Ehrenfest method. [39] In particular, the states $\left|\psi_{j}(t, \tau)\right\rangle$ are expanded as

$$
\left|\psi_{j}(t, \tau)\right\rangle=\sum_{\alpha=1}^{N} c_{j, \alpha}(t, \tau)\left|g_{\alpha}(t, \tau)\right\rangle,
$$

where $\left|g_{\alpha}(t, \tau)\right\rangle$ is a Gaussian wavepacket whose center moves along the classical trajectory of $H_{1}$ until the delay time $\tau$ and with the average Hamiltonian $\bar{H}$ from then on. The expansion coefficients $c_{j, \alpha}(t, \tau)$ satisfy the time-dependent Schrödinger equation

$$
i \hbar \mathbf{S} \dot{\mathbf{c}}_{j}=\left\{\begin{array}{cc}
\left(\mathbf{H}_{1}-i \hbar \mathbf{D}\right) \mathbf{c}_{j}, & 0<t^{\prime}<\tau \\
\left(\mathbf{H}_{j}-i \hbar \mathbf{D}\right) \mathbf{c}_{j}, & \tau<t^{\prime}<\tau+t
\end{array},\right.
$$

where $\mathbf{H}_{j}$ is the Hamiltonian matrix, $\mathbf{S}$ is the overlap matrix, and $\mathbf{D}$ the nonadiabatic coupling matrix defined by their matrix elements in the Gaussian basis:

$$
\begin{array}{ll}
H_{\alpha \beta}(t, \tau) & :=\left\langle g_{\alpha}(t, \tau)\left|\hat{H}_{j}\right| g_{\beta}(t, \tau)\right\rangle, \\
S_{\alpha \beta}(t, \tau) & :=\left\langle g_{\alpha}(t, \tau) \mid g_{\beta}(t, \tau)\right\rangle, \\
D_{\alpha \beta}(t, \tau) & :=\left\langle g_{\alpha}(t, \tau) \mid \dot{g}_{\beta}(t, \tau)\right\rangle .
\end{array}
$$

In the Gaussian dephasing representation (GDR), ${ }^{[40]}$ one runs classical trajectories as in the original dephasing representation, but uses them only to guide the Gaussian basis functions $g_{\alpha}(t, \tau)$. The time dependence of the expansion coefficients $c_{j, \alpha}$ are obtained by solving the time-depen- dent Schrödinger equations (17), and finally, the fidelity amplitude is evaluated as

$$
f_{\mathrm{GDR}}(t)=\mathbf{c}_{1}(t, \tau)^{\dagger} \mathbf{S}(t, \tau) \mathbf{c}_{0}(t, \tau) .
$$

Note that if the Gaussian basis $g_{\alpha}(t, \tau)$ is large enough, the Gaussian dephasing representation is not a semiclassical approximation; indeed, it should converge to the exact quantum answer as the Gaussian basis approaches completeness. (Beware, however, of various numerical issues due to nonorthogonality of the basis, etc. ${ }^{[37-40]}$ )

Fig. 4 demonstrates the accuracy of the Gaussian dephasing representation on the time-resolved stimulated emission spectrum of pyrazine, showing that the Gaussian dephasing representation with 576 basis functions yields a correlation function and spectrum which are basically indistinguishable from the exact quantum analogs, unlike the fully converged dephasing representation, which does contain a semiclassical error.

As for the computational cost of the Gaussian dephasing representation, the number of trajectories required is much smaller than in typical semiclassical methods, and can be even smaller than in the original dephasing representation since in the GDR, the trajectories carry with them Gaussian basis functions, which play a smoothing role similar to the cells in the cellular dephasing representation. The most expensive part per trajectory of the GDR is the evaluation of the Hamiltonian matrix elements $H_{\alpha \beta}$ : using, e.g. a local harmonic approximation for the potential requires the Hessian of the potential energy, but one can often get away only with a linear expansion that only necessitates the gradient, which is already needed for propagating the trajectories. The final contribution to the cost is solving the time-dependent Schrödinger equation, which scales as $O\left(N^{3}\right)$, i.e. cubically with the number of trajectories, which is
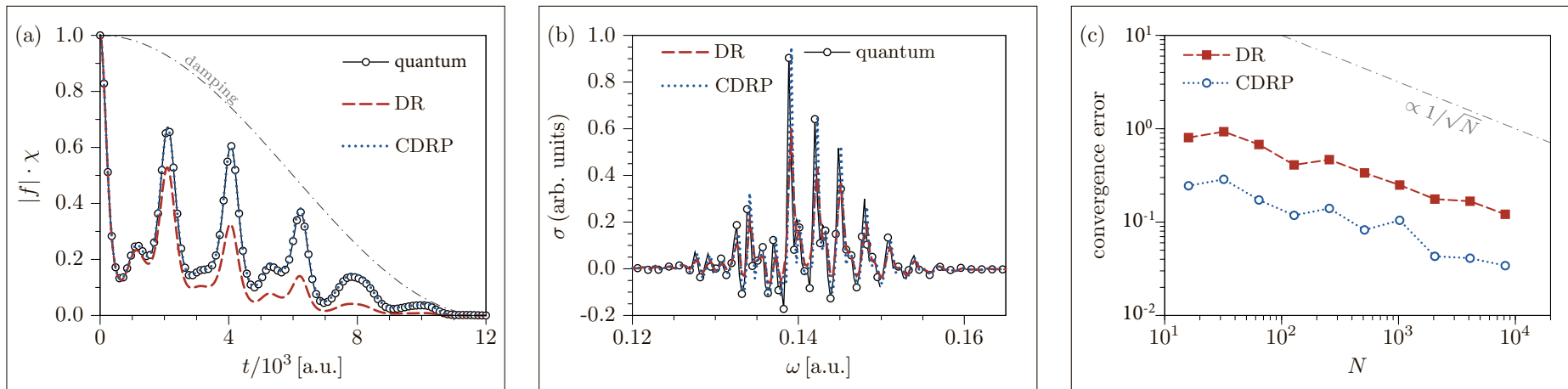

Fig. 3. Time-resolved stimulated emission in the pyrazine $S_{0} / S_{1}$ model from ref. [36]. Initial state is the ground state of the $S_{0}$ surface and the delay time between pump and probe pulses is $\tau=2 \times 10^{3}$ a.u. $\approx 48 \mathrm{fs}$. Comparison of the exact quantum result, the original dephasing representation (DR), and the cellular DR with a prefactor (CDRP). (a) Time correlation function (already multiplied by a damping function indicated by a dash-dotted line). (b) Corresponding spectrum. (c) Convergence error (relative $L^{2}$ norm error) of the damped correlation function as a function of the number of trajectories N. (Adapted from ref. [36].) 

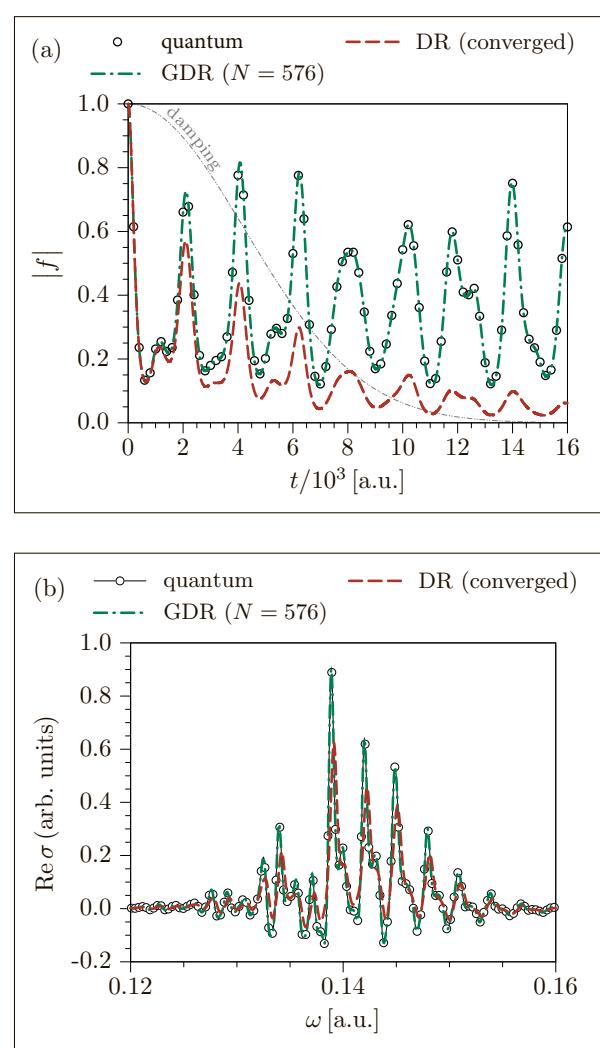

Fig. 4. Time-resolved stimulated emission in the same pyrazine $S_{0} / S_{1}$ model as in Fig. 3 . Comparison of the exact quantum result, the original dephasing representation (DR), and the Gaussian DR (GDR) based on 576 trajectories only. (a) Time correlation function. (b) Corresponding spectrum obtained as a Fourier transform of the correlation function multiplied with a damping function indicated in panel (a) by a gray dash-double-dotted line. (Adapted from ref. [40].)

much worse than the linear $O(N)$ scaling of the dephasing representation, whose $N$ trajectories are independent. On the other hand, and in particular in on-the-fly $a b$ initio applications, the cost of the electronic structure, especially the Hessian, becomes easily so high that the cubic scaling of the solution of the Schrödinger equation may still be negligible if the number of trajectories is below a few hundred.

\section{Conclusions and Outlook}

All methods that we have discussed so far express correlation functions needed in time-resolved spectra calculations in terms of interfering contributions from classical trajectories. This common feature of all the discussed methods makes possible their implementation together with an onthe-fly $a b$ initio evaluation of electronic structure, possibly turning them into an automated and almost 'black box' tool for analyzing ultrafast spectra without the necessity of a tedious construction of global or semi-global potential energy surfaces.
The accuracy of the approximate methods, can be, of course, improved in other ways than using Gaussian basis methods. Recently, ${ }^{[41]}$ we have described an arbitrary-order expansion of the Feynman path integral representation of the correlation functions (4) or (6), of which the first-order expansion yields the dephasing representation, while the zeroth-order expansion gives the static classical limit, ${ }^{[10]}$ which itself is very useful. It turns out that already the second order expansion would correct most of the shortcomings of the dephasing representation in typical chemical systems (which are neither exactly harmonic, nor chaotic); unfortunately, the most straightforward implementation is very inefficient.

Another important aspect of ultrafast spectra not captured by the methods reviewed here is the common presence of nonadiabatic and spin-orbit couplings between various electronic states contributing to the spectra, which give rise to internal conversion or intersystem crossings between various states. If weak, these processes lead only to the broadening of the spectra, but, if strong, they can completely change the spectral line shapes. To address this issue, we have generalized the dephasing representation to the setting of coupled electronic states and obtained the multiplesurface dephasing representation ${ }^{[42]}$ that can capture the major consequences of the nonadiabatic or spin-orbit couplings on ultrafast electronic spectra. This method can and has been combined with an on-the-fly $a b$ initio evaluation of energies, forces, and nonadiabatic couplings.

Besides the efficiency and ease of onthe-fly evaluation of electronic structure, the trajectory-based methods for evaluating ultrafast spectra have another advantage, probably the most important of all. They provide an intuitive picture of the dynamics, which is much easier to decipher than, e.g. a 30-dimensional wavefunction in the case of pyrazine. While the simpler picture of course does not have to be quantitatively correct $100 \%$ of the time, we have been time and again surprised by the qualitative correctness of the semiclassical result.

\section{Acknowledgements}

This research was supported by the Swiss NSF with the grant number 200020150098 and within the NCCR Molecular Ultrafast Science and Technology (MUST), and by the ERC Consolidator Grant Project No. 683069 MOLEQULE. The author would like to thank all the former and current members of the Laboratory of Theoretical Physical Chemistry at EPFL for their contributions to the research reviewed in this article, Julien Roulet for translating the manuscript from Latex to Microsoft Office Word, and Sergey Antipov for helpful comments.

Received: April 11, 2017
[1] E. J. Heller, Acc. Chem. Res. 1981, 14, 368

[2] E. J. Heller, J. Chem. Phys. 1975, 62, 1544

[3] S. Mukamel, 'Principles of nonlinear optical spectroscopy', 1st ed., Oxford University Press, New York, 1999.

[4] S. Mukamel, J. Chem. Phys. 1982, 77, 173.

[5] T. Gorin, T. Prosen, T. H. Seligman, M Žnidarič, Phys. Rep. 2006, 435, 33.

[6] W. T. Pollard, S.-Y. Lee, R. A. Mathies, J. Chem. Phys. 1990, 92, 4012.

[7] N. E. Shemetulskis, R. F. Loring, J. Chem Phys. 1992, 97, 1217.

[8] J. M. Rost, J. Phys. B 1995, 28, L601.

[9] Z. Li, J.-Y. Fang, C. C. Martens, J. Chem. Phys. 1996, 104, 6919

[10] S. A. Egorov, E. Rabani, B. J. Berne, J. Chem Phys. 1998, 108, 1407.

[11] S. A. Egorov, E. Rabani, B. J. Berne, J. Chem Phys. 1999, 110, 5238

[12] Q. Shi, E. Geva, J. Chem. Phys. 2005, 122, 064506.

[13] H. M. Pastawski, P. R. Levstein, G. Usaj, J. Raya, J. Hirschinger, Physica A 2000, 283, 166.

[14] C. Petitjean, D. V. Bevilaqua, E. J. Heller, P. Jacquod, Phys. Rev. Lett. 2007, 98, 164101.

[15] T. Zimmermann, J. Vaníček, J. Chem. Phys. 2010, 132, 241101

[16] T. Zimmermann, J. Vaníček, J. Chem. Phys. 2012, 136, 094106

[17] T. Zimmermann, J. Vaníček, J. Chem. Phys. 2012, 137, 22A516.

[18] B. Li, C. Mollica, J. Vaníček, J. Chem. Phys 2009, 131, 041101

[19] T. Zimmermann, J. Ruppen, B. Li, J. Vaníček, Int. J. Quant. Chem. 2010, 110, 2426.

[20] M. Wehrle, M. Šulc, J. Vaníček, Chimia 2011, 65,334

[21] M. Šulc, J. Vaníček, Mol. Phys. 2012, 110, 945

[22] E. J. Heller, J. Chem. Phys. 1981, 75, 2923.

[23] W. H. Miller, J. Chem. Phys. 1970, 53, 3578

[24] W. H. Miller, J. Phys. Chem. 2001, 105, 2942.

[25] M. F. Herman, E. Kluk, Chem. Phys. 1984, 91 , 27.

[26] J. Vaníček, Phys. Rev. E 2004, 70, 055201.

[27] J. Vaníček, Phys. Rev. E 2006, 73, 046204.

[28] E. Zambrano, A. M. Ozorio de Almeida, Phys. Rev. E 2011, 84, 045201(R).

[29] W. H. Miller, F. T. Smith, Phys. Rev. A 1978, 17 , 939.

[30] L. M. Hubbard, W. H. Miller, J. Chem. Phys. 1983, 78, 1801.

[31] C. Mollica, J. Vaníček, Phys. Rev. Lett. 2011, 107,214101

[32] E. J. Heller, J. Chem. Phys. 1991, 94, 2723.

[33] V. S. Filinov, Nucl. Phys. B 1986, 271, 717.

[34] N. Makri, W. H. Miller, Chem. Phys. Lett. 1987, $139,10$.

[35] A. R. Walton, D. E. Manolopoulos, Mol. Phys. 1996, 87,961

[36] E. Zambrano, M. Šulc, J. Vaníček, J. Chem. Phys. 2013, 139, 054109.

[37] M. Ben-Nun, T. J. Martínez, Adv. Chem. Phys. 2002, 121, 439.

[38] G. A. Worth, M. A. Robb, I. Burghardt, Faraday Discuss. 2004, 127, 307.

[39] D. V. Shalashilin, J. Chem. Phys. 2010, 132, 244111

[40] M. Šulc, H. Hernandez, T. J. Martínez, J. Vaníček, J. Chem. Phys. 2013, 139, 034112.

[41] J. Vaníček, D. Cohen, Phil. Trans. R. Soc. A 2016, 374, 20150164.

[42] T. Zimmermann, J. Vaníček, J. Chem. Phys. 2014, 141, 134102 . 